\title{
Does Chronic Remyelination Occur for All Spared Axons after Spinal Cord Injury in Mouse?
}

\author{
John C. Gensel, ${ }^{2,4}$ Akshata A. Almad, ${ }^{1,3,4}$ Jessica K. Alexander, ${ }^{2,3,4}$ David L. Schonberg, ${ }^{1,3,4}$ and Richa B. Tripathi ${ }^{1,4}$ \\ Departments of ${ }^{1}$ Neuroscience and ${ }^{2}$ Molecular Virology, Immunology, and Medical Genetics, ${ }^{3}$ Neuroscience Graduate Studies Program, and ${ }^{4}$ The Center for \\ Brain and Spinal Cord Repair, The Ohio State University College of Medicine, Columbus, Ohio 43210 \\ Review of Lasiene et al. (http://www.jneurosci.org/cgi/content/full/28/15/3887)
}

Spinal cord injury is a long-lasting, debilitating condition that affects $\sim 250,000$ people in the United States. Spontaneous functional recovery after human spinal cord injury is limited because of severe axonal damage and poor axonal regeneration. Hence, a major area of focus has been to preserve and optimize the conductivity of the spared but demyelinated axons that survive the initial insult.

Myelin is indispensable for efficient saltatory conduction in axons and the maintenance of their healthy state. Therefore, a number of treatments after experimental spinal cord injury have strived to achieve remyelination by transplanting cells capable of forming myelin and ensheathing axons. Although these strategies improved functional recovery when delivered acutely after injury, more chronic application produced fewer noticeable improvements (Eftekharpour et al., 2008). This raises questions about whether demyelination exists in axons capable of some activity chronically after injury, and whether conduction deficits in these axons contribute to functional impairments. Before therapies can be advanced, these basic questions need to be resolved.

A recently published study by Lasiene et al. (2008) examined the myelin status

Received June 4, 2008; revised July 7, 2008; accepted July 7, 2008. Correspondence should be addressed to John C. Gensel, 705 Biomedical Research Tower, 460 West 12th Avenue, Columbus, OH 43210. E-mail: gensel.1@osu.edu.

DOI:10.1523/JNEUROSCI.2533-08.2008

Copyright $\odot$ 2008 Society for Neuroscience $\quad$ 0270-6474/08/288385-02\$15.00/0 and simulated functional properties of spared axons chronically after injury. They studied actively transporting rubrospinal tract (RST) axons in C57BL/6 mice at select time points after contusive spinal cord injury. The authors defined actively transporting axons by Fluoro-Ruby and biotinylated dextran amine (BDA) labeling [Lasiene et al. (2008), their Fig. 1 (http://www.jneurosci.org/cgi/content/ full/28/15/3887/F1)]. Several methods were used to examine myelin indices, including comparing g-ratios (axon diameter/ total fiber diameter) and measurements of internode length on anterogradely labeled axons. As stated by the authors, it is often difficult to investigate demyelinating pathologies using standard myelin stains [Lasiene et al. (2008), their Fig. 3H (http:// www.jneurosci.org/cgi/content/full/28/15/ 3887/F3)]. Therefore, they used the distribution of the paranodal protein contactinassociated protein (CASPR) and the voltage-gated $\mathrm{K}_{\mathrm{v}} 1.2$ ion channel normally found in the juxtaparanodal regions as measures of myelin integrity.

Lasiene et al. (2008) first investigated acute demyelination after mouse spinal cord injury. Two weeks after injury, irregular CASPR and $\mathrm{K}_{\mathrm{v}} 1.2$ labeling was detected along Fluoro-Ruby-traced rubrospinal axons [Lasiene et al. (2008), their Fig. $2 A$ (http://www.jneurosci.org/cgi/ content/full/28/15/3887/F2)]. This abnormal distribution suggests that myelin is disrupted and/or lost, which was verified in toluidine blue semithin plastic sec- tions, a common method for examining demyelination [Lasiene et al. (2008), their Fig. 2B-D (http://www.jneurosci.org/cgi/ content/full/28/15/3887/F2)]. The results were confirmed by decreased MBP (myelin basic protein) mRNA expression acutely after spinal cord injury [Lasiene et al. (2008), their Fig. $2 E$ (http://www.jneurosci. org/cgi/content/full/28/15/3887/F2)].

Because acute demyelination is well documented after spinal cord injury, the majority of the study by Lasiene et al. (2008) addressed chronic demyelination in mice 12 weeks after injury. Distribution of CASPR and $\mathrm{K}_{\mathrm{v}} 1.2$ proteins was not diffuse or irregular in Fluoro-Ruby-labeled axons at this time, although they displayed reduced internodal lengths compared with control axons [Lasiene et al. (2008), their Fig. 3E,F (http://www.jneurosci.org/cgi/ content/full/28/15/3887/F3) and Fig. 4 (http://www.jneurosci.org/cgi/content/full/ 28/15/3887/F4)]. The presence of shorter internodes is typical of remyelinated axons. Remyelination was confirmed using a second anterograde tracer, BDA, coupled with electron microscopy [Lasiene et al. (2008), their Fig. 5D (http://www.jneurosci.org/cgi/ content/full/28/15/3887/F5)]. Trends toward thinner myelin profiles (increases in the ratio of axon diameter to total fiber diameter) were detected for axons spared after spinal cord injury compared with controls [Lasiene et al. (2008), their Fig. 5E (http:// www.jneurosci.org/cgi/content/full/28/15/ 3887/F5)]. This reduced myelin thickness was significantly different only for smaller- 
diameter axons $(\leq 2 \mu \mathrm{m})$. These data provide evidence that there is complete remyelination of RST axons 12 weeks after spinal cord injury. Hence, Lasiene et al. (2008) conclude that there is no evidence for chronic demyelination in spared axons after spinal cord injury in mice.

In contrast to the findings by Lasiene et al. (2008), both demyelination and remyelination have been shown to occur chronically after spinal cord injury in rats (Totoiu and Keirstead, 2005). In that study, demyelination was prevalent acutely and then decreased over time (up to 10 weeks after injury). The initial decrease in demyelination coincided with a progressive increase in remyelination starting 1 week after injury and continuing until 17 weeks. Surprisingly, there was a secondary wave of demyelination that continued until 64 weeks, at which point little remyelination was occurring. Thus, remyelination in rats is thought to be incomplete at later time points after spinal cord injury. Because little is known about chronic demyelination after mouse spinal cord injury, it would be valuable to determine whether the findings by Lasiene et al. (2008) are representative of all chronic time points and/or whether mouse injury also produces a dynamic biphasic demyelination as in rats.

To determine whether decreased internodal lengths and myelin thickness could affect conduction properties of remyelinated axons, Lasiene et al. (2008) performed computer simulations based on a double-cable axon model. Using data obtained from Fluoro-Ruby- and BDAlabeled axons, simulation studies revealed a $32 \%$ decrease in the average conduction velocity (CV) of injured RST axons compared with controls [Lasiene et al. (2008), their Fig. 6A (http://www.jneurosci.org/ cgi/content/full/28/15/3887/F6)]. Simulations considering only axons between 2 and $4 \mu \mathrm{m}$ showed a $21 \%$ decrease in the average CV compared with controls [Lasiene et al. (2008), their Fig. 6 B (http:// www.jneurosci.org/cgi/content/full/28/ 15/3887/F6)]. To determine whether myelin thickness was affecting conduction velocities, Lasiene et al. (2008) repeated the simulation using the same myelin thickness for spared and control axons. This yielded similar results, indicating that decrease in conduction velocities was likely because of changes in internodal length and not thinner myelin. Lasiene et al. (2008) suggest that this $21 \%$ decrease is unlikely to affect locomotion in mice but may have greater functional consequences for larger animals in which axons conduct over greater distances.
The novelty of the study by Lasiene et al. (2008) was the examination of myelin indices on axons anterogradely labeled with Fluoro-Ruby or BDA. For these tracers to be taken up, axons must be capable of active transport. The authors creatively used this property to evaluate demyelination in spared, functional axons (those capable of active transport). This approach may provide insight into the potential for remyelination strategies to improve axonal function. However, a recent study by Ong et al. (2008) suggests that these tracers may have preferentially labeled larger-diameter axons of the rubrospinal tract. Using a combination of conventional toluidine blue histology and diffusion magnetic resonance imaging techniques, Ong et al. (2008) showed that the mean axonal diameter of RST axons in intact C57BL/6 mice was 1.16 and $1.4 \mu \mathrm{m}$, respectively. This is in contrast to Lasiene et al. (2008), who reported that the majority of Fluoro-Ruby-labeled RST axons were between 2.0 and $4.0 \mu \mathrm{m}$ in diameter in uninjured and injured animals. Although a few differences exist between the two studies (spinal cord level, tissue processing, and age of the animals), the average diameter of uninjured myelinated axons in rats is also smaller $(1.75 \mu \mathrm{m}$ in diameter) than those labeled by Lasiene et al. (2008) (Fehlings and Tator, 1995). This suggests that in the study by Lasiene et al. (2008), the tracers may have preferentially labeled larger-diameter axons.

To avoid the caveats of relying on active transport, Lasiene et al. (2008) used a lentiviral reporter, pCMV-GFP (plasmid cytomegalovirus-green fluorescent protein), to examine the myelin status of passively labeled axons [Lasiene et al. (2008), their Fig. 7 (http://www.jneurosci.org/ cgi/content/full/28/15/3887/F7)]. Comparing the number and/or diameter of passively versus actively labeled axons in controls could help determine whether size-specific labeling properties exist for Fluoro-Ruby and BDA labels. If, indeed, differences exist between the labeling techniques, smaller-diameter axons may have been underrepresented in their analysis. If so, the extent of myelin pathology may have been greater than reported, because only the small/medium-diameter axons $(<2 \mu \mathrm{m})$ had significantly reduced myelin thickness after injury (Lasiene et al., 2008). Furthermore, their report of an insignificant decrease in mean axonal diameter of FluoroRuby-labeled axons after spinal cord injury may have resulted from oversampling larger-diameter axons (Lasiene et al., 2008).

Although preferential labeling of larger-diameter axons may have influenced some parameters, such a bias would not likely influence predicted conduction velocities. Lasiene et al. (2008) predicted a $21 \%$ decrease in conduction velocity based on metrics gathered from anterogradely labeled axons. This is consistent with real-time recordings from the RST after rat spinal cord injury (van de Meent et al., 1996). Lasiene et al. (2008) also examined CASPR and $\mathrm{K}_{\mathrm{v}} 1.2$ labeling on passively labeled axons 12 weeks after injury. Morphologically normal axons demonstrated spatially compact CASPR and $\mathrm{K}_{\mathrm{v}} 1.2$ labeling. Diffuse CASPR and $\mathrm{K}_{\mathrm{v}} 1.2$ labeling was only detected on morphologically dystrophic axons. This suggests that intact axons of the RST were remyelinated at this time point.

Experiments performed by Lasiene et al. (2008) clearly show that rubrospinal tract axons capable of active transport are not demyelinated 12 weeks after injury. The authors undergo several steps to demonstrate that this population of axons has the histological, molecular, and functional properties of myelinated axons. This is significant because it suggests therapies aimed at increasing chronic remyelination after injury fail, in part, because few axons exist which are demyelinated at chronic states. Hence, it would be valuable to determine whether these data are consistent with other spinal cord tracts, other chronic time points, and other labeling techniques. These considerations should be examined in future studies to further understand the status of chronic demyelination after mouse spinal cord injury.

\section{References}

Eftekharpour E, Karimi-Abdolrezaee S, Fehlings MG (2008) Current status of experimental cell replacement approaches to spinal cord injury. Neurosurg Focus 24:E19.

Fehlings MG, Tator CH (1995) The relationships among the severity of spinal cord injury, residual neurological function, axon counts, and counts of retrogradely labeled neurons after experimental spinal cord injury. Exp Neurol 132:220-228.

Lasiene J, Shupe L, Perlmutter S, Horner P (2008) No evidence for chronic demyelination in spared axons after spinal cord injury in a mouse. J Neurosci 28:3887-3896.

Ong HH, Wright AC, Wehrli SL, Souza A, Schwartz ED, Hwang SN, Wehrli FW (2008) Indirect measurement of regional axon diameter in excised mouse spinal cord with q-space imaging: simulation and experimental studies. Neuroimage 40:1619-1632.

Totoiu MO, Keirstead HS (2005) Spinal cord injury is accompanied by chronic progressive demyelination. J Comp Neurol 486:373-383.

van de Meent H, Hamers FP, Lankhorst AJ, Buise MP, Joosten EA, Gispen WH (1996) New assessment techniques for evaluation of posttraumatic spinal cord function in the rat. J Neurotrauma 13:741-754. 